\title{
Spatial Dependence of the Sheath Power Transmission Factor in DIII-D*
}

\author{
A. H. Futch, G. F. Matthews ${ }^{t, D}$ D. Buchenauer $\ddagger$, D. N. Hill, and G. D. Porter
}

\author{
Lawrence Lvermore National Laboratory \\ Livermare, CA, 94550
}

\begin{abstract}
The spatial dependence of the power transmission factor, $d$, associated with an ionelectron pair passing tivrough the sheath at the DIII-D divertor plate has been determined by sweeping the edge plasma across Langmuir probe detectors. Our results show that d decreases from the classically expected value of eight near the low density edge of the scrape-off-layer plasma to values less than unity at the peak of the profile.
\end{abstract}

\section{MASTER}

This woik was performed under the auspices of the U. S. Department of Energy by the Lawrence Livermore National Laboratory under Contract W-7405-ENG-48.

${ }^{+}$Culham Laboratory, Abingdon, Oxon, United Kingdom ¥Sandia National Laboratories, Livermore, CA. 


\section{Introduction}

In this paper we re-examine previous measurements of $\delta$ where values in the range of 2 to 3 were reported (Ref. 1). The thearetical value of $\delta$ expected from Stangeby's formula (Ref. 2)

$$
\delta=\frac{2 T_{i}}{T_{e}}+\frac{2}{1-\gamma_{e}}-0.5 \ln \left[2 \pi \frac{m_{e}}{m_{i}}\left(1+\frac{T_{1}}{T_{a}}\right)\left(1-\gamma_{e}\right)^{-2}\right],
$$

is approximately eight if the secondary electron emission coefficient, $\boldsymbol{\gamma}_{e}$, is zero. Inclusion of current flow or secondary electron emission increases the value of $\delta$, and therefore, the discrepancy between theory and the reported measurements becomes larger. The authors of Ref. 1 suggest that the anomalously low measured values of $\delta$ in DIII-D results from an efiective ion collecting area which in much larger than the projected geometric arra. This explanation was suggested by experiments on DITE (Ref. 3), where mer surements using a tilting array of llush-mounted Langmuir probes showed the effective ion collection area to be much larger than the projected geometric aree if the r.ngle between the divertor tiles and the magnetic field is less than $10^{\circ}$.

To determine $\delta$, we equate the expression for the power flow to the DIII-D divertor tiles as calculated from the Langmuir probe data (Ref. 4) to IR camera measurements of the beat fux (Ref. 5), PIR, deposited on divertor tiles. Since the power flow as calculated from \& Langanuir probe is $P_{L P}=\delta I_{\text {ant }} T_{0} \sin \theta / A_{\perp}$, where $\theta=$ $\theta_{B}+\theta_{\varepsilon}$ is the angle between the floor tiles and the magnetic field direction, and $\theta_{c}$ 
is the deviation from the horizontal plane of any particular foor tile and $\theta_{B}=B_{4} / B_{4}$, the expression for $\delta$ becomes

$$
\delta=\frac{P_{\text {IR }} A_{\perp}}{\mathrm{I}_{\text {mat }} T_{1} \sin \theta}
$$

In Eq. (2), $I_{2 n}$ is the measured ion saturation current, and $A_{\perp}$ is the projected area of the probe perpendicular to the plasma flow.

In our re-examination of the DIII-D data, we find a large time dependence of $\delta$ during a shot. This observation eliminates the suggestion that the anomalous low value of $\delta$ results from an effective area much larger than the geometric area since the field angle does not vary significantly during the shot. We relate the large changes in $\delta$ during a shot to the spatial dependence of $\delta$ as the edge plasma p.ofile is swept across the Langmuir probes, cod suzgest that the anomalously low ralues of $\delta$ results from localized gas densities produced by recyeling of the divertor current. 


\section{Experiment}

The experiment arrangement shown in Fig. 1 is the same as that discussed in Ref. 1. Although 21 Langmuir probes have now been installed across the divertor floor to provide profile data without the necessity of sweeping, the data analyzed here is limited to the previous configuration with Langmuir probes installed in only two locations. The design of the Langrauir probes are similar to those used on JET and are domed to produce a projected area, $A_{\perp}$, which is insensitive to the field angle over the range of field angles typical of the DIII-D divertor $\left(0 \rightarrow 5^{\circ}\right)$. The IRTV camera system installed near the top of the DIII-D vacuum tank views the divertor floor and Langmuir probes as shown in Fig. 1. Results presented here are from the two probes located near the outer strike point at major radii of $1.707 \mathrm{~m}$ and $1.735 \mathrm{~m}$. In some of the data the strike point was swept over the probes several times during the discharge. In other shots the strike point varied unintentionally early in time during the discharge and more slowly as equilibrium was approached. The angle of the magtetic field with respect to the divertor tile is approximately constant during an individual shot even though sweeping occurs, since it is the radial and not the vertical field which is altered during a sweep. The data has been corrected for the difference in $\sin \theta$ between the two probe locations as well as for the difference between shots. For example, $\theta$ had average values of $3.8^{\circ}$ at $R=1.707 \mathrm{~m}$ and $4.65^{\circ}$ at $R=1.735 \mathrm{~m}$ during the time period of 1 to $2.4 \mathrm{~s}$ for the discharge of Shot 66837. In analyzing the IRTV data, we also correct for the fact that the tile on which the IRTV data was recorded is tilted at an angle of $0.3^{\circ}$ stch that the deposited power is increased. 


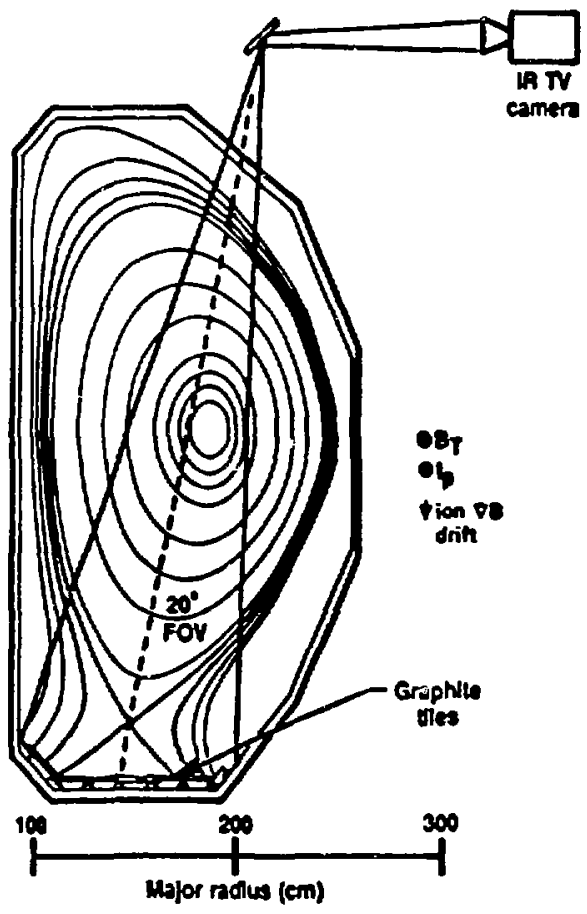

Fis. 1. Sehematic view of DIII-D showing the divertor floor tiles containing the Langmuir probes. 


\section{Experimental Results}

Plasma parameters and associated magnetic field parameters are shown in Fig. 2 as a function of time for Shot 66837 which is a typical non-swept shot that we have analyzed. The various parameters plotted in this figure are defined in the captions for the figure. Figure 3 shows a time-dependent plot of $\delta$ for the Langmuir probe at $1.735 \mathrm{~m}$. Early in the shot $(0.5$ to $1.3 \mathrm{~s}) \delta$ is approximately 6 , but then decreases to values below 2 as the strike point approaches the detector (see curve $f$ of Fig. 2 where the distance between the detector and the otrike point is plotted). At approximately $2.2 \mathrm{~s}$, the neutr: beams turn on, the strike point begins to move away from the Iangmuir probe, and $\delta$ begins to increase back to the 8 to 10 range. Values above 8 appear to be associated with either the presence of enhanced visible Bremsstrahlung (curve $g$ of Fig. 2) or the presence of ELMs. Very large values of $\delta$ during ELM activity probably result from radiative power being detected by the IRTV camera instead of power lost by an ion-electron pair passing through the sheath potential. We have therefore limited our discussion in the rest of this paper to non $H$-mode data wbich we believe to be not unduly influenced by radiation. We bave also excluaded from consideration any data points which appear to be in the private flux region since any particle flow for this region is very small and the resulting error is likcly to be large.

The time dependent values of $n_{e}$ and $T_{e}$ are given in Fig. 4(a) and Fig. $4(b)$ for the Langmuir probe at 1.735 m. Figure $5(\mathrm{a})$ shows calculated values of $\delta$ versus $n_{e} \sqrt{T_{e}}$ for the non $\mathrm{H}$-mode data of Shot 66837 at the radial location of $1.735 \mathrm{~m}$. Figure 5 (b) shows the corresponding curve for the detector at $1.707 \mathrm{~m}$. We have chosen to plot $\delta$ versus $n_{e} \sqrt{T_{e}}$ since this quantity should be proportional to the particle flux striking 


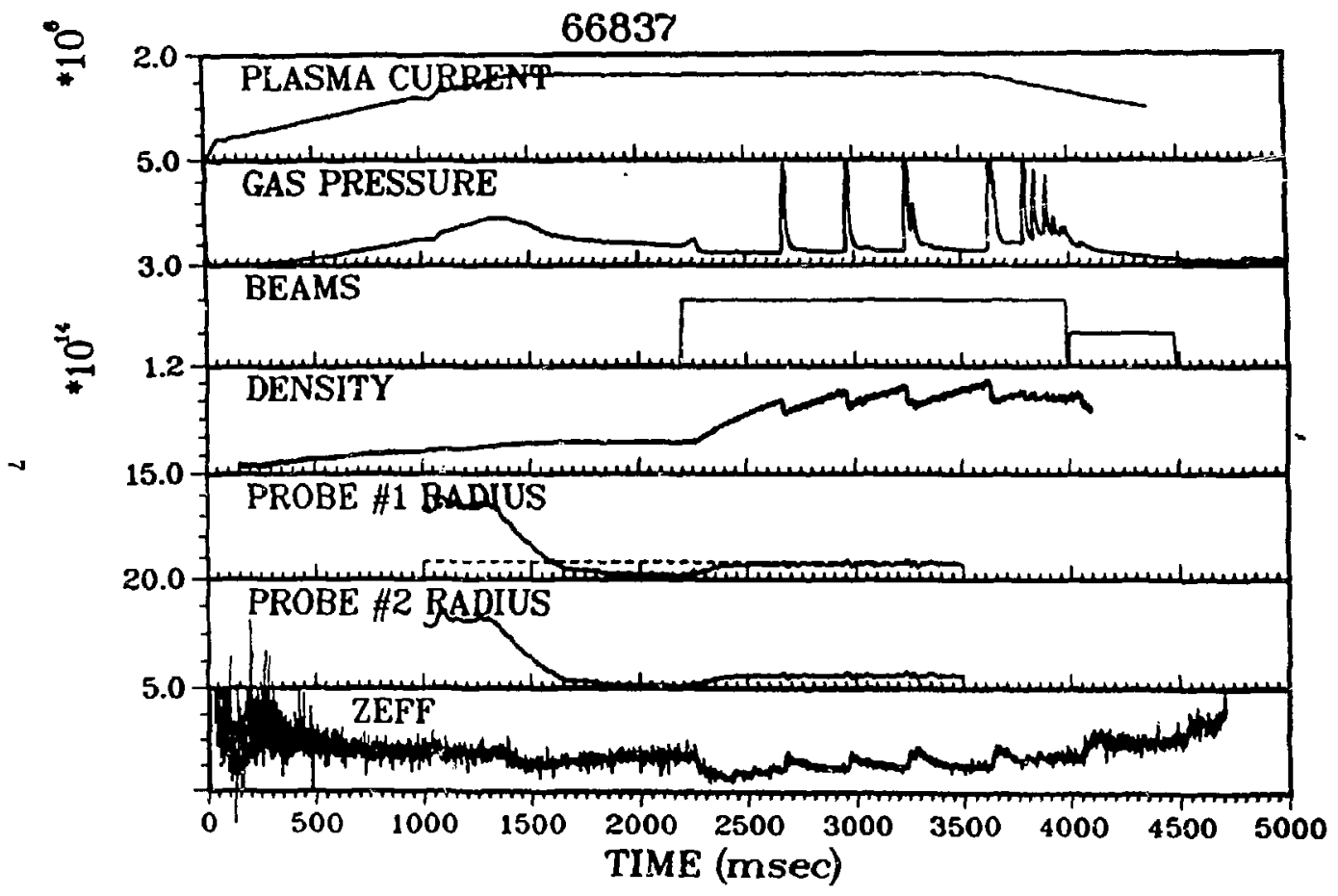

Fig. 2. Phamm porameters and associsted magnetic field parometers for Shot 66837 . Curves a through $g$ are the plasma current in amperes, relative neutral gas pressure, number of neutral beams, plasma density in $\mathrm{em}^{-3}$, Langmuir probe \#1 radius relative to the outer strike point in cm, Langrmuir \#2 radiun relative to the outer strike point in $\mathrm{cm}$, effective 2 for the plasma. 


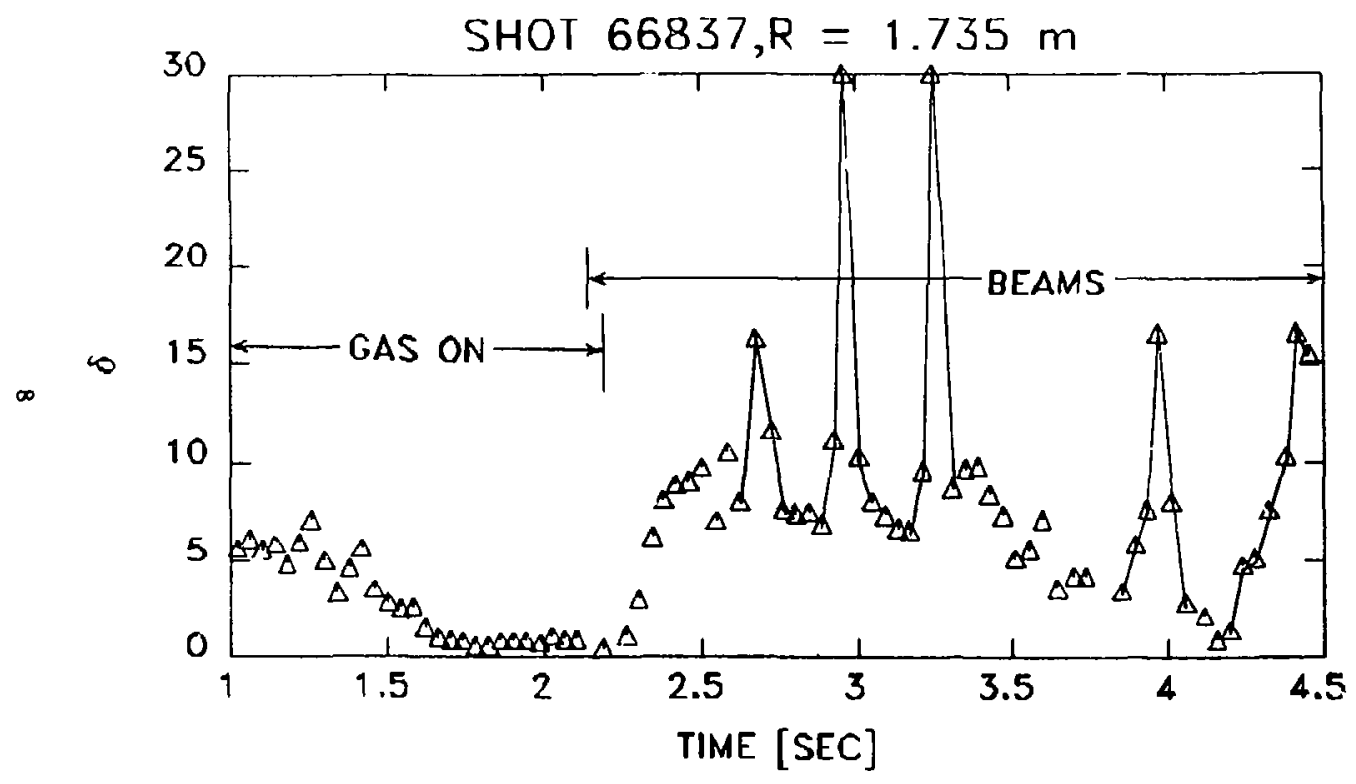

Fig. 3. Time dependent plot of the sheath power transmission factor, $\delta$ for Shut 66837 it $R=1.735 \mathrm{~m}$. 

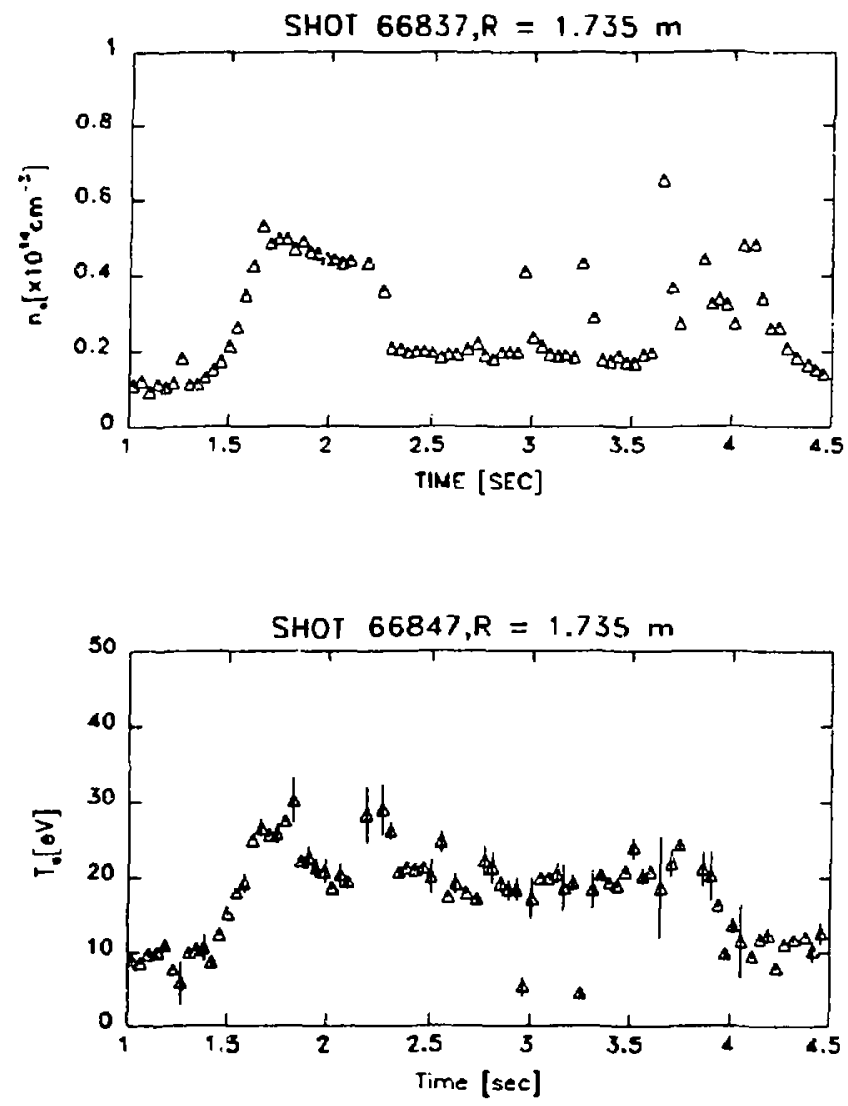

Fig. 4. Time dependent plots of the electron density (a) and the electron temperature (b) for Shot 66837. 

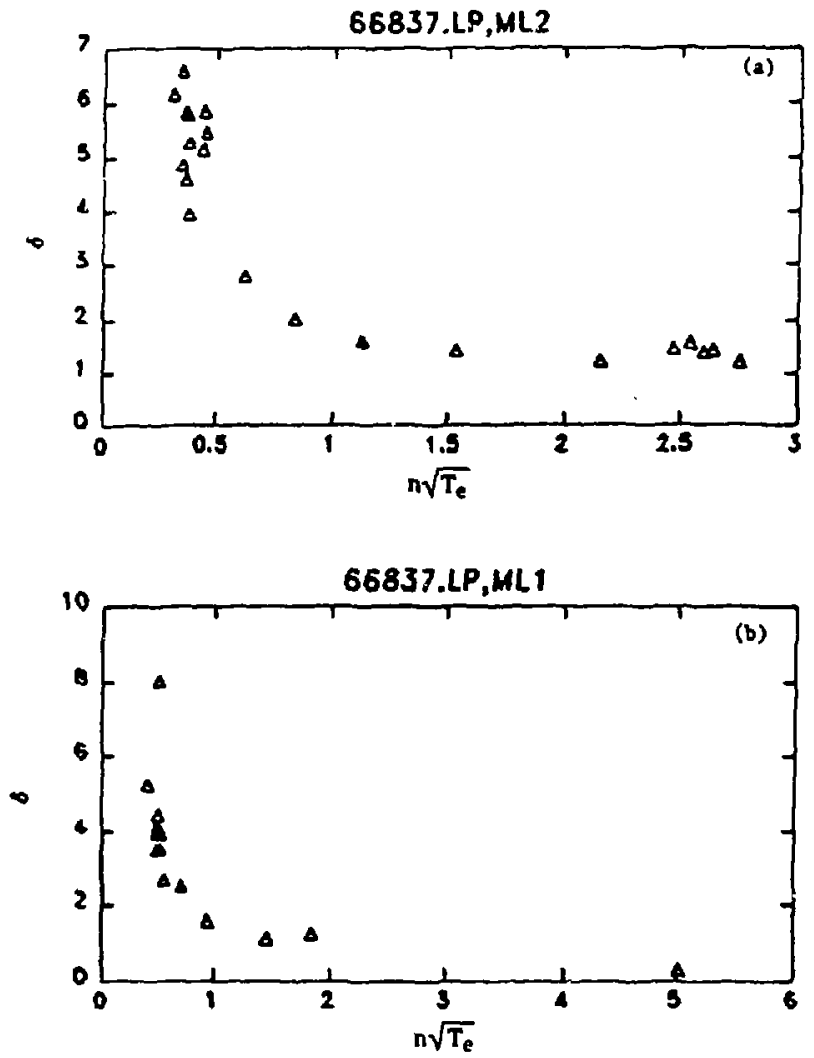

Fig. 5. The power transmission factor, $\delta$, versus $n \sqrt{ } T_{0}$ for 5 hot 66837 at (a) $R=1.735 \mathrm{~m}$ and at (b) $R=1.707 \mathrm{~m}$. 
the detector, and based on DEGAS modelling, we have speculated that this quartity is proportional to the local gas density. Both of these curves show $\delta$ to be close to the expected value of eight near the edge of the scrape-off-layer profile where $n \sqrt{T_{e}}$ is umall and decreasing to values in the range of two or lower at the peak of the profile. We find this behavior to be consistent for all the shots wave analyzed. Figures 6 and 7 show similar results for two additional shots; these are Shots 66835 and 66840 , respectively. The results for all of these shots are plotted on the same graph in Fig. 8.

Shot 66796 is one of the shots where the strike points are swept across the two Langmuir probes three times during the shot. This is also the shot which was analyzed in Ref. 1. Figure 9 shows the time dependence of plasma parameters and associated magnetic field parameters. These parameters are defined in the captions for this shot. The value of $\delta$ as a function of time is given in Fig. 10.

In Fig. 11, $\delta$ is plotted versus the parameter $n_{\mathbb{*}} \sqrt{T}$, for Langmuir probes located at both radial locations $(1.707 \mathrm{~m}$ and $1.735 \mathrm{~m})$. The plasma for this shot is produced by the injection of deuterium beams onto a bydrogen plasma, i.e., $\mathrm{H}_{2}$ gas is used to initiate the discharge. Although the $\mathrm{D}^{+}$beams are ramped up to $4.5 \mathrm{MW}$, the discharge remains in the $\mathbf{L}$-mouie for the entire shot duration. The uncertainty in $\delta$ observed for Shot 66796 seems somewhat greater than for the non-swept shots. In addition, for $n_{\varepsilon} \sqrt{T_{a}}$ in the range from $1 \times 10^{14}$ to $3 \times 10^{14}, \delta$ is also somewhat higher. This observation is probably best illustrated in the composite plot of Fig. 12. The explanation for the increased noise and somewhat higher values of $\delta$ could be selated to the fact that neutral beams up to $4.5 \mathrm{MW}$ are present ind sigaificant radiative bursts are indicated by the bolometer. The spred detector also shows a high level of carbon present at this time. 

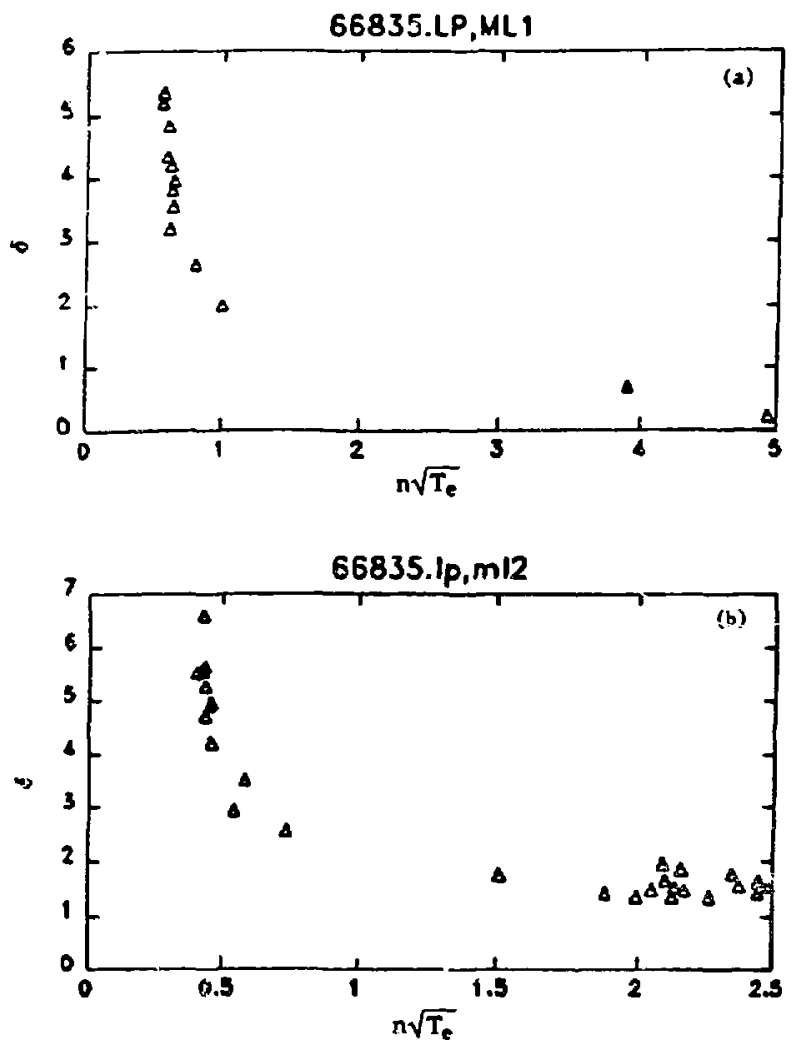

Fig. 6. The powep transmission factor, $\delta$, versus $n \sqrt{T_{e}}$ for Shot 66835 at (a) $R=1.707 \mathrm{~m}$ and at (b) $R=1.735 \mathrm{~m}$. 


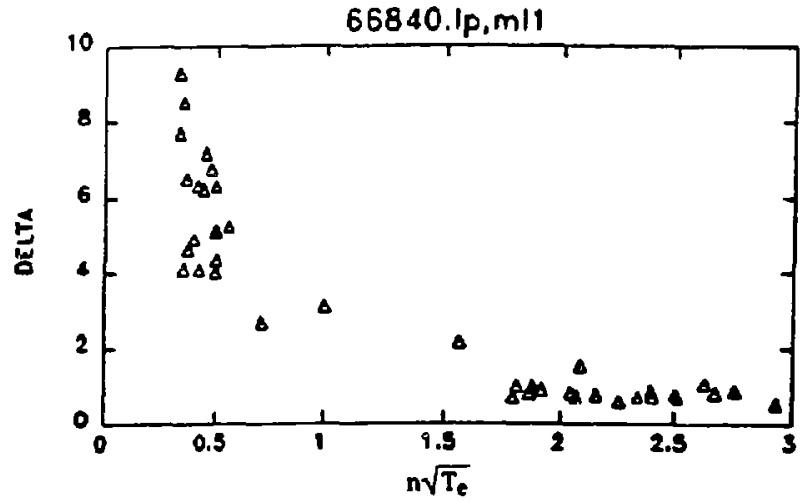

Fic. 7. The power transmission factor, $\delta$, versus $n \sqrt{T_{e}}$ for Shot 66840 at $R=1.707 \mathrm{~m}$. 


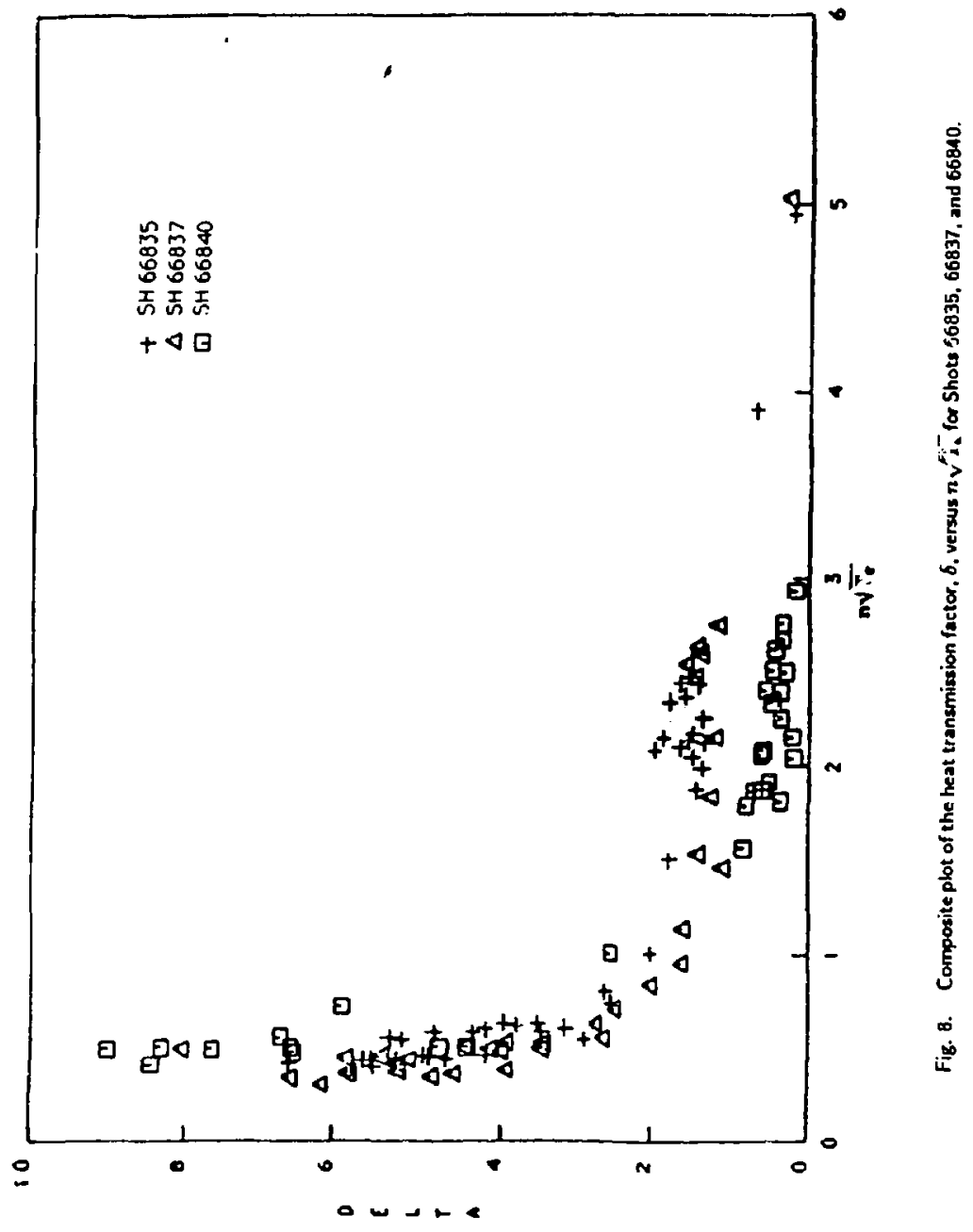




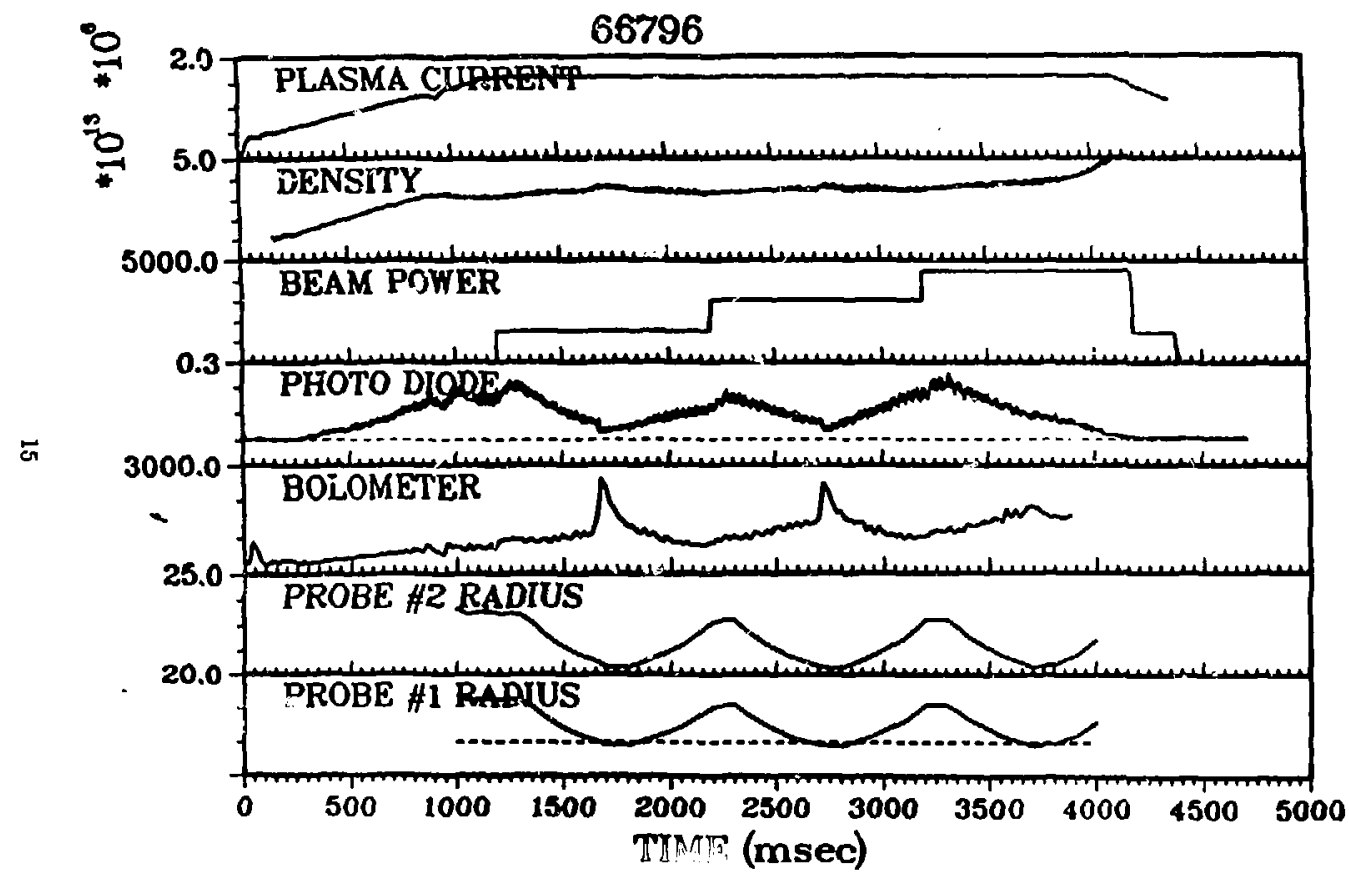

Fig. 9. Plaxma parameter and associated magnetic field parameters for Shot 66796 . Curves a through $g$ are the plasma current in amperes, the plasma density in $\mathrm{cm}^{-3}$, the neutral beam power in kilowatts, a photo diode signal which is proportional to $\mathrm{Ha}_{a}$ emissions, solometer wignal which measures the radiative power enitted, Langmuir probe \#1 racius relative to the outer strike point, Langmuir probe $\$ 2$ radius relative to the outer strike point. 


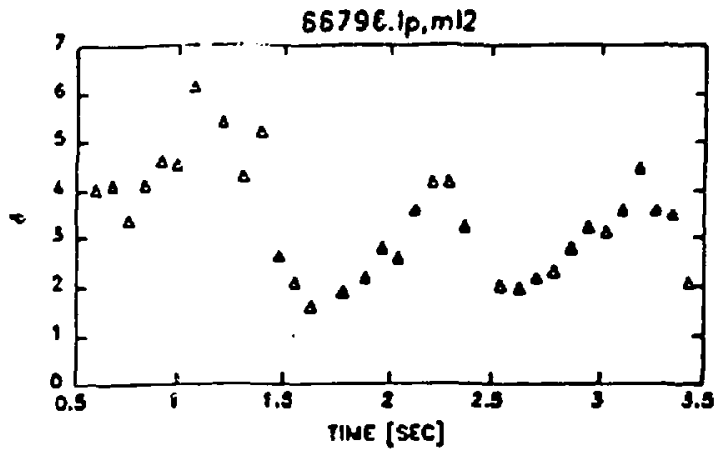

Fig. 10. Time dependent plot of the sheath power transmission factor, $\delta$, for Shot 66796 at $R=1.735 \mathrm{~m}$. 

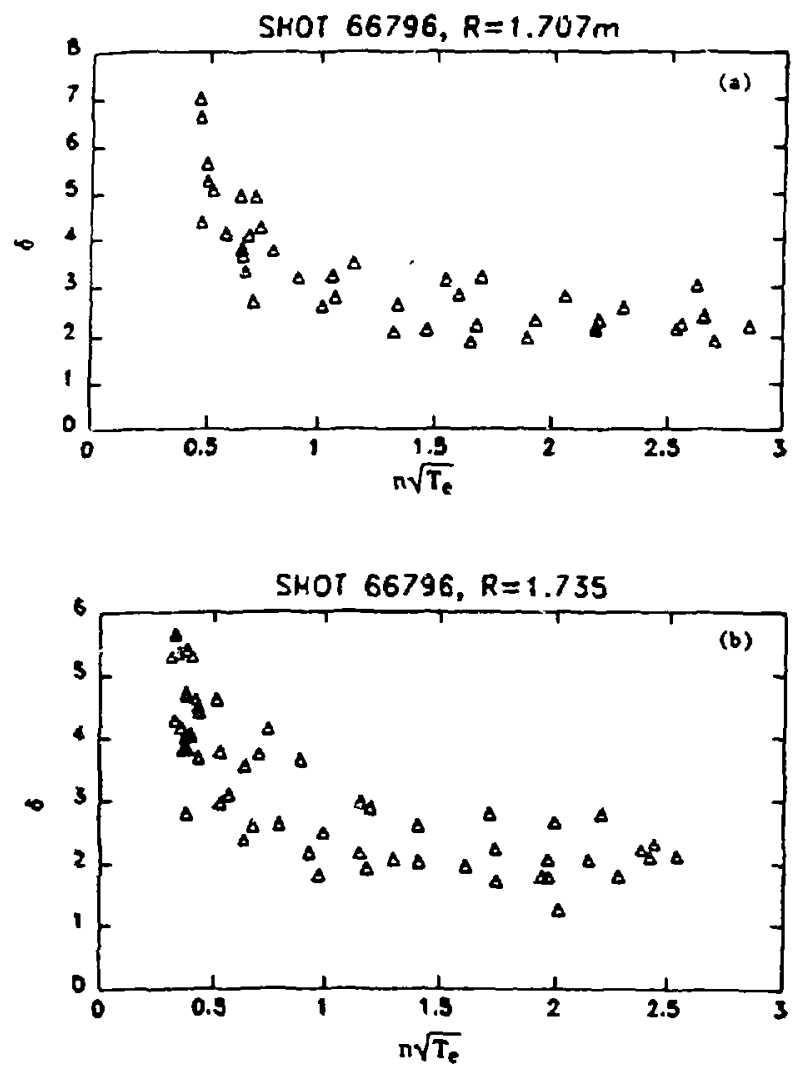

Fig. 11. The power transmission factor, $\delta$, versus $n \sqrt{T_{e}}$ for Shot 66796 at (*) $R=1.707 \mathrm{~m}$ and at (b) $R=1.735 \mathrm{~m}$. 


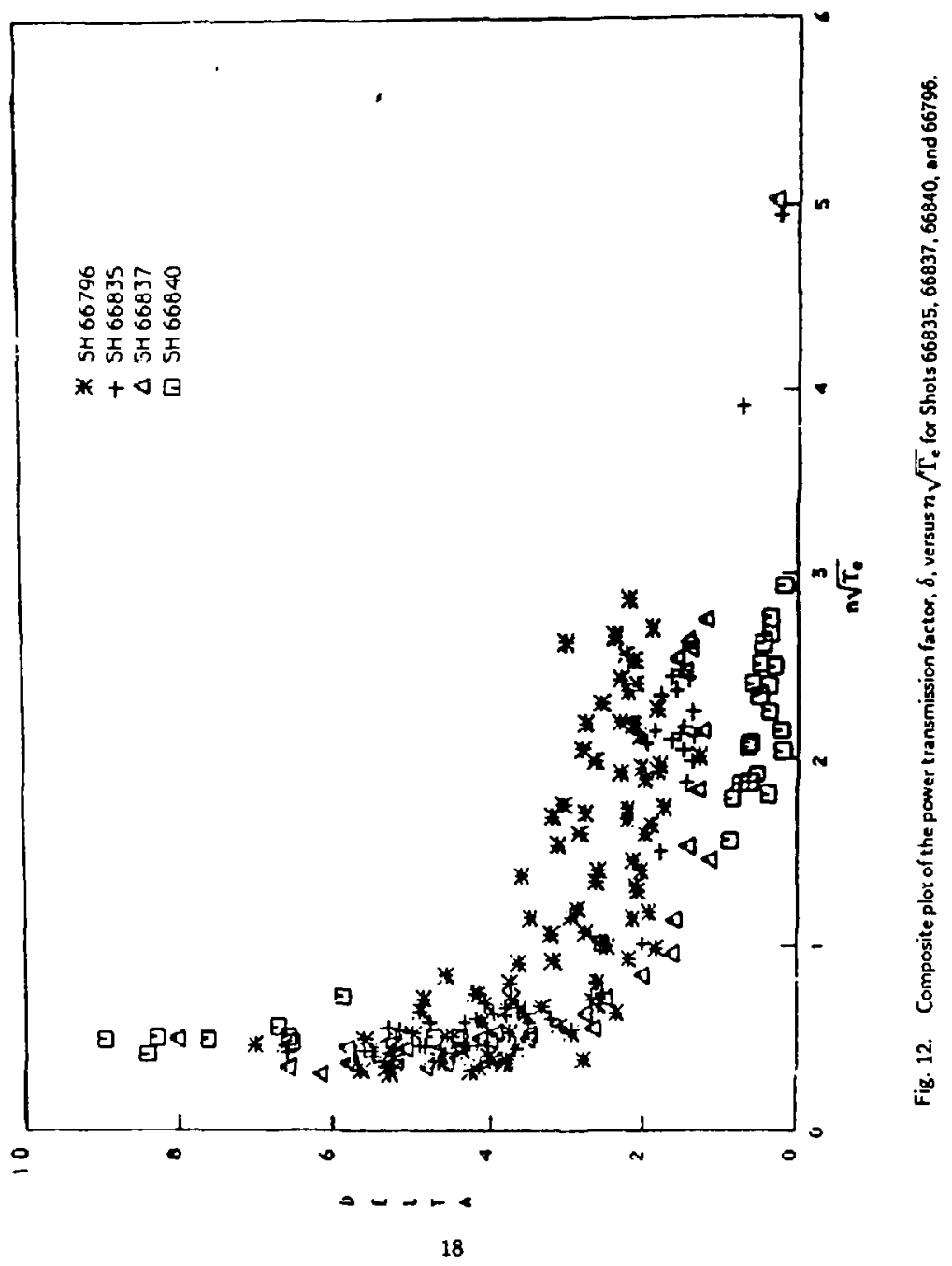




\section{Possible Interpretation of Observed Spatial Dependence of $\delta$}

Existing theories for the electrostatic sheath pocential that forms between a plasma and a wall to prevent a net flow of current solves Poisson's equation in the absence of particle sources or sinks except at the boundaries (Refs. 6-9). Using a numerical model, Choduca studied the effect of a magnetic field on the ccllisionless electrostatic sheath (Ref. 10). Daybelge and Bein bave also studied the collisionless

electrostatic sheath in the presence of a uniform magnetic field baving a sanall angle of incidence with respect to the surface (Ref. 11). An important feature of their results is that for negative wall potentials the scale length of the sheath in front of an absorbing wall is the ion Larmor radius, whereas for positive wall potentials this length reduces to the electron Larmor radius.

For tokamek plasmas such as DIII-D, the wall potential is negative with respect to the plasma. Therefore, according to Ref. 11, the scale length for the sheath potential perpendicular to the surface is the ion Larmor radius. Although this distance is small for the magnetic fields of DIII-D we will show that for a one-for-one recycling of plasma to neutral atoms at the divertor tiles as modeled by DEGAS, local neutral gas densities are sufficiently large, that significant atomic collisions occur within the sheath potential and the collisionless assumptions of existing theories or numerical models are not valid.

A large number of atomic collisions within the potential sheath scale length will influence the heat transmission factor $\delta$ in at least twc ways. First, the potential will be different from that calculated by collisionless theory wid second, ions that undergo 
collisions within the potential sheath will no longer arrive at the wall surface with an energy of $e \phi+2 T_{i}^{0}$ where $T_{i}^{0}$ is the ion temperature prior to being accelerated by the sheath. From the se sond effect, we expect $\delta$ to decrease from the classical value of 8 to $\sim 2 i_{\varepsilon}$ for sufficiently larger neutral gas pressures. Here we have assumed $Z$ is unity and that the ion $T_{i}$ after the collision is negligible. Of course, coulomb collisions could eventually lower $T_{e}$, but t be large Langmuir probe size makes spatial measurements of $T_{e}$ within the sheath impossible.

Although we have no local measuremeuts of gas pressure on the divertor tiles, the profile dependence of $\delta$ is consistent with the speculation that $\delta$ is reduced by a sufficiently large gas density near the plasma wall interface. We note that it is possible to measure a profile dependence of the floating potential and we have analyzed data from one of the shots to look for changes in the potential as the strike point morres acroas the Langmuir probes. We find that the floating potential decreases as the edge profile is swept across the Langmuir probe and will discuss this result later in this section.

We now attempt to justify our claim thet a collisionless potential theory is not valid for the divertor tiles in DIII-D and that a lower power transmission factor is expected for sufficiently high neutral gas pressures. Although we have no direct measurements of the local gas pressure at the strike points, DEGAS modelling of gas densities in several locations along the DIII-D vacuum walls have been consistent with measurements of gas densities at these locations (factor of 2 agreement). For our estimate of the gas pressure under the strike point we use the DEGAS results from SEP2790 which is a run for a standard L-mode discharge. The atomic neutral density number under the peak of the outer strike point was $2.7 \times 10^{15}$ atoms $/ \mathrm{cm}^{3}$.

From the measured charge exchange $\sigma_{\mathrm{cx}}$ and elastic scatterings $\sigma_{2}$ cross-sections given in Fig. 13(a) and (b), respectively, we obtain approximate values of $5 \times 10^{-15} \mathrm{~cm}^{2}$ and $1 \times 10^{-14} \mathrm{~cm}^{2}$ for a $10 \mathrm{eV} \mathrm{H}^{+}$ion. These crose-sections are observed to be slowly 


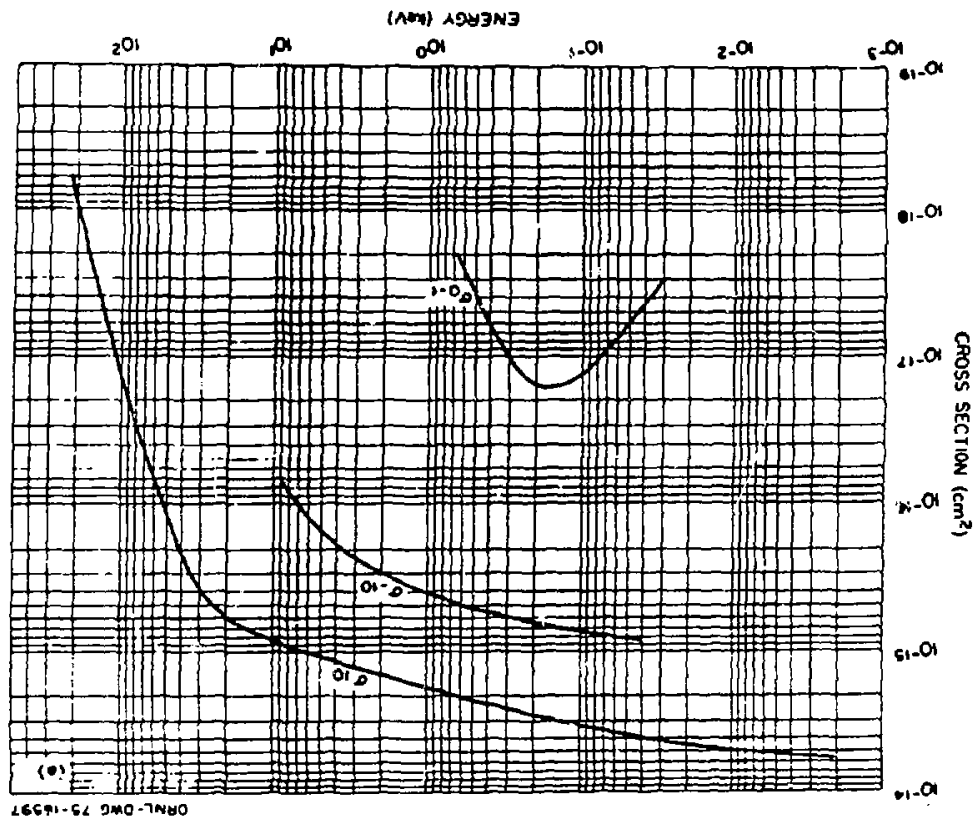




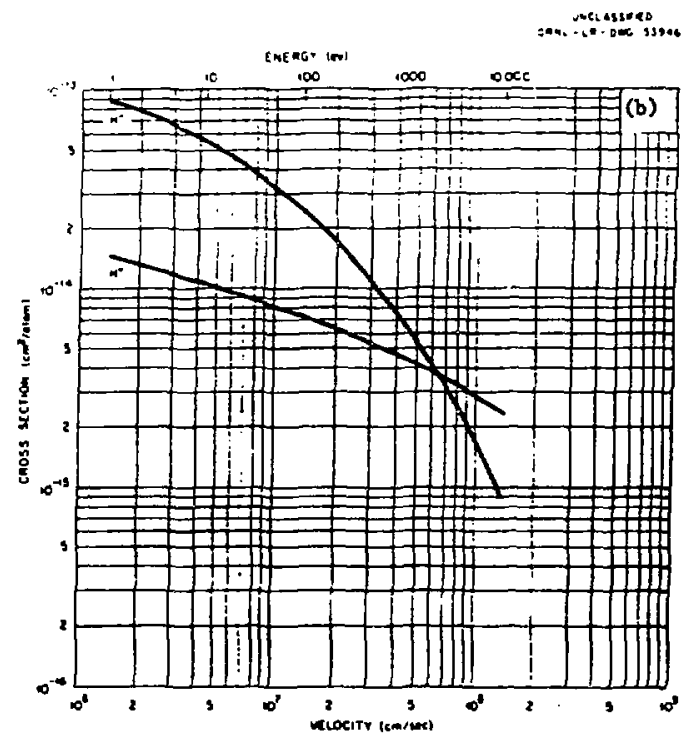

Fig. 13. Charge exchange (a) and elastic seattering cross section (b) from Ref. 12. (Sheet 2 of 2) 
varying at this energy. A neutral $D^{\circ}$ atom will penetrate a distance $\lambda=\left(n_{0} \sigma_{c \infty}\right)^{-1}=$ $5.7 \mathrm{~cm}$ before being converted into an ion by a charge exchange collision. Therefore the neutral density is essentially constant orer the potentinl scale length. Electron ionization will not significantly reduce this distance since the electron ionization crosssection is smaller than the resonance charge exchange cross-section. Calculating the mean free path for an ion to lose energy by either charge exchange or elastic scattering as $\left(n_{e} \cdot s\right)^{-1}$, we find the mean free path to be $0.07 \mathrm{~cm}$ and $0.04 \mathrm{~cm}$, respectively. Either one of these collisions will reduce the energy the ion gained in falling through the potential sheaths and lead to a reduction in $\delta$. Including both of these processes, we obtain a combined mean free path $\lambda_{t}$ of

$$
\frac{1}{\lambda_{t}}=\frac{1}{\lambda_{0}}+\frac{1}{\lambda_{2}}=40 \mathrm{~cm}^{-1},
$$

or $\lambda_{t}=2.5 \times 10^{-2} \mathrm{~cm}$. The ion Larmor radius is

$$
\rho=\frac{2 \times 10^{2}}{B} \sqrt{\mathrm{E}}=3.2 \times 10^{-2} \mathrm{~cm}
$$

for an ion energy of $10 \mathrm{eV}$ and a magnetic field of $2 \mathrm{~T}$. An ion moving along the field line will be in the potential sheath region for a aistance of $\rho / \sin \theta \approx 0.5$ cm for $\theta=4$ degrees which is a typical value of $\theta$ at the divertor otrike point. Although a finite temperature perpendicular to the magnetic field can shorten this distance, we conclude that the mean free path for atomic collision is less than the seale length for the potential sheath and therefore ar ion passing through the potential sheath has a good probability of losing energy as a result of collisions before reaching the wall surface. This energy loss will result in a smaller value for the power transmission factor. We have also pointed out that the inclusion of collisions in the sheath theory 
could also change the magnitude of the potential itself which would lead in turn to a lower transmission factor if the sheath potential is smaller.

For a non-magnetic plasma, the sheath potential can be obtained from the exponential dependence of the I-V characteristic for the Langmuir probe above the foating potential, $V_{f}$. In a strongly magnetized plasma, the I-V characteristic is observed to be non-exponential for voltages above $\mathrm{V}_{f}$. In addition plasma fuctuations further distorts the I-V characteristic especially above the floating potential making probe measurements of the sheath potential unreliable. However, we have obtained measurements of the flonting potential and also observed a correlation between the power transmission factor, $\delta$, and the fonting potential.

Figure 14 shows the measured floating potential, $V_{f}$, versus $n \sqrt{T_{e}}$ for shot 66796 at $R=1.735 \mathrm{~m}$ which is the swept shot discussed earlier. The time dependence of both the floating potential and $\delta$ is exhibited in Fig. 15, and in Fig. 16, the foating potential is plotted versus $\delta$. The straight line in the figure is a least squares fit to the experimental data. This observed correlation is inconsistent with existing models for the power transmission factor (Ref. 6-11, 13) where $\delta$ is independent of the floating potential. Here again, a possible explanation for this paradax is that both $v_{f}$ and $\delta$ are functions of another parameter which we suggest is the local neutral gas pressure. The large decrease in $\delta$ as the profile is swept across the delestor requires both a decrease in the sheath potential as well as a decrease in the kinetic energy of the ions and electrons prior to the potential shesth drop. This is additional evidence that tive local gas pressure has an effect on the sheath potential as well as the measured effect on the floating potential. 


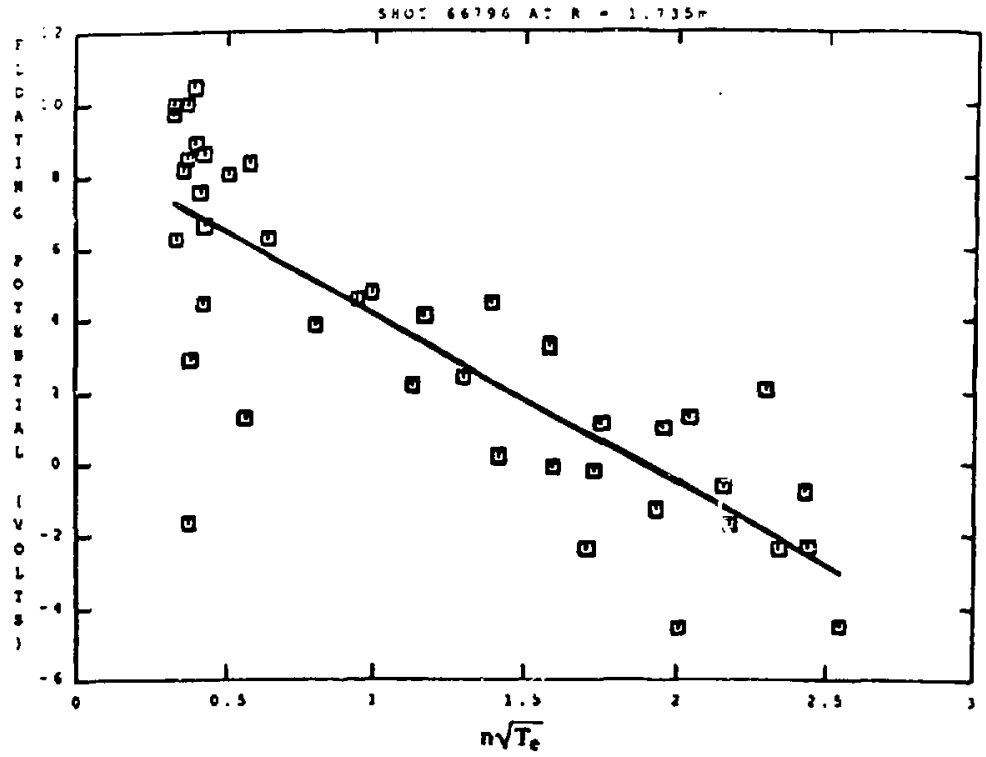

Fig. 14. The dependence of the flating potential, $\because$, , as a function of $n \sqrt{T_{*}}$ for Shat 66796 at $R=1.735 \mathrm{~m}$, the araight line is a least square fit of the equation $V_{f}=$ $a(0)+a(1) n \sqrt{T_{a}}$ through the data points where $a(0)=8.75$ and $a(1)=-4.63$. 


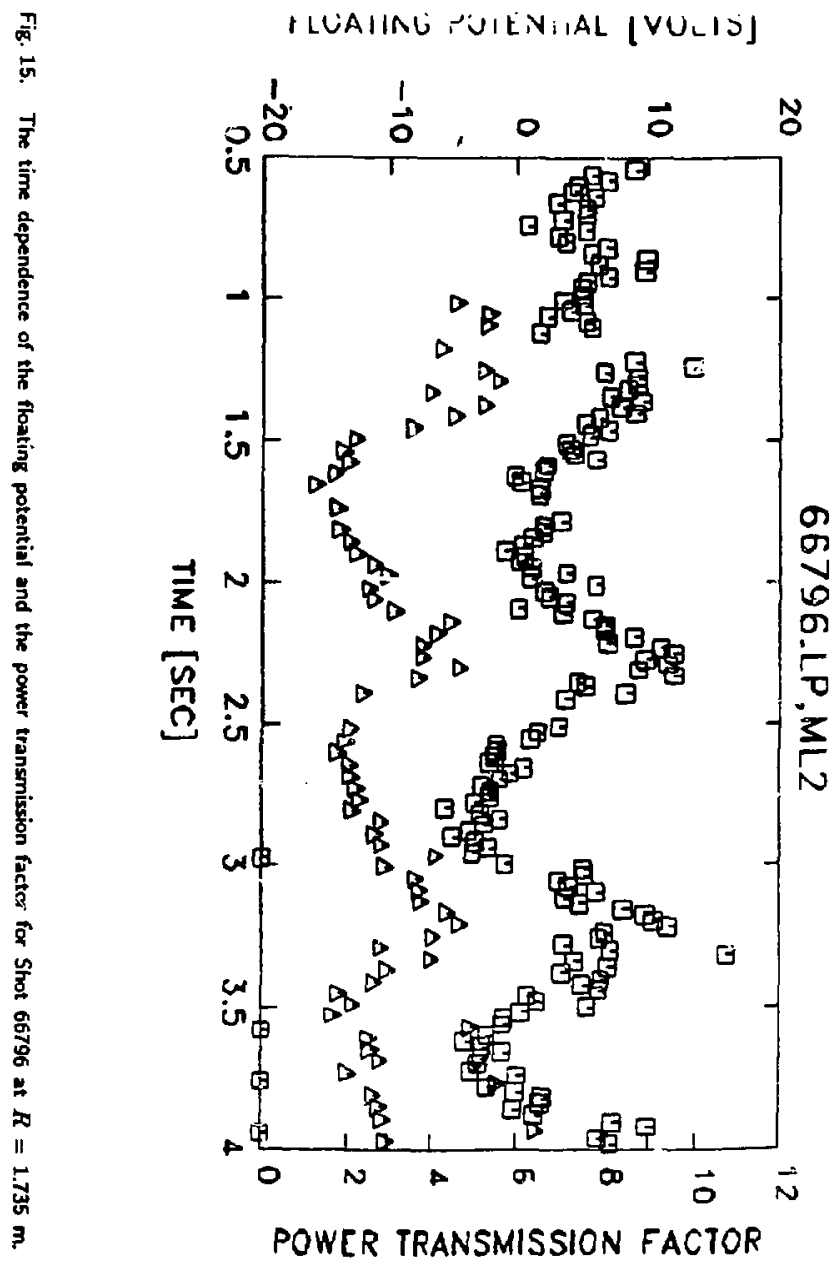




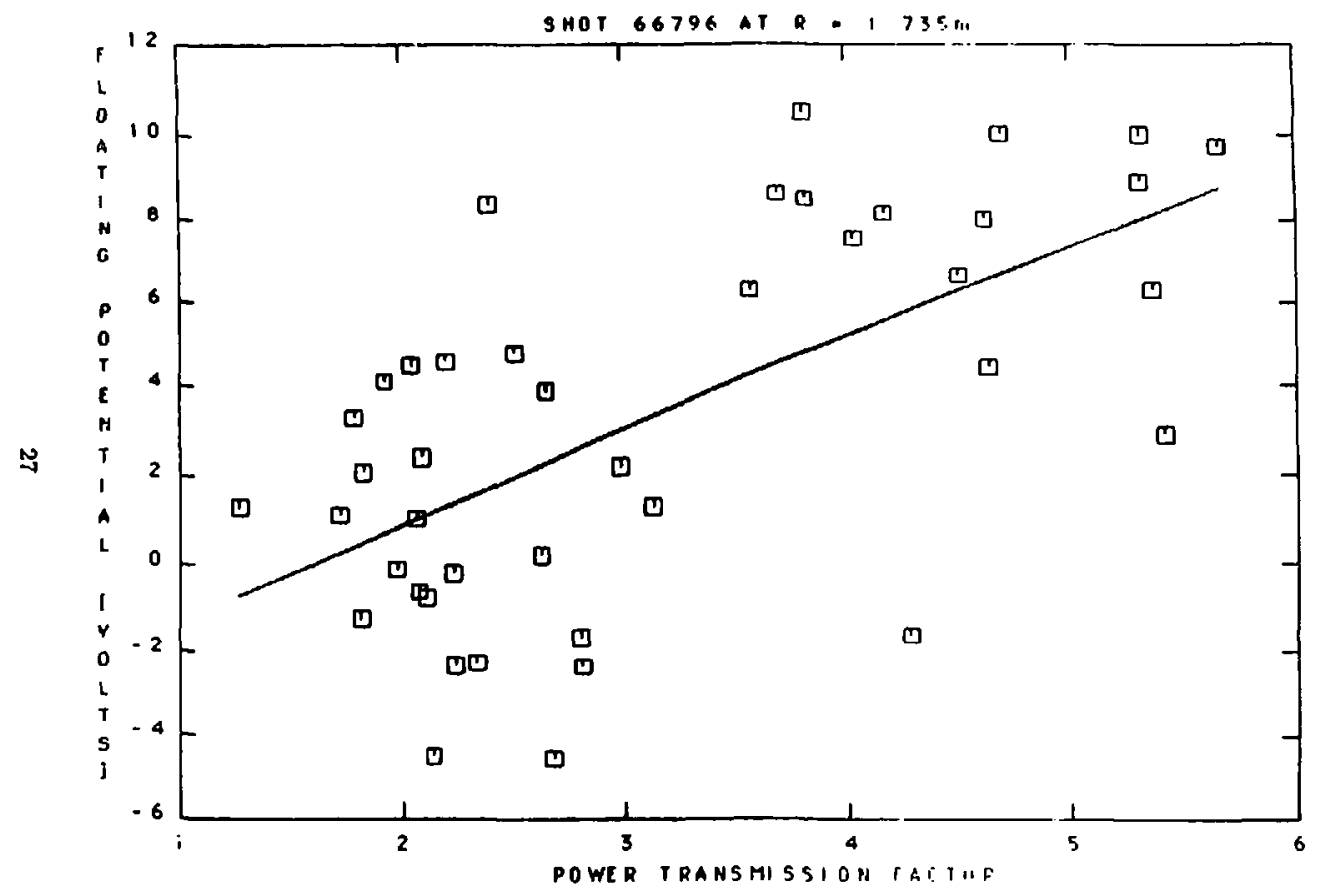

Fig. 15. The floating potential versus the power tranamission factor for Shot 66796 at $R=1.735 \mathrm{~m}$. The straight line is a least squares fit of the equation $V_{f}=a(0)+a(1) \delta$ through the data points where $a(0)=-3.46$ and $a(1)=2.17$. 


\section{References}

1. G.F. Matthews, D. Buchenauer, D.N. Hill, and M.A. Małdavi, "The Sheath Power Transmission Factor in DIII-D," General Atomics Report GA-A20337, October 1990 submitted to Plasma Physics and Controlled Fusion.

2. P.C. Stangeby in "Physics of Plasma-Wall Lteractions in Controlled Fusion" Plenum Press, New York, 1986 (D.E. Post and R. Behrisch, eds.) pp. 41-97.

3. G.F. Matti'ewg, G.M. McCracken, P.C. Stangeby, "Plasma Physica and Controlled Fusion," to be published.

4. D. Buchenauer, W.L. Hsu, J.P. Smith, D.N. Hill, Rev. Sci. Instru. 61, 2873, (1990).

5. D.N. Bill, R. Ellis, W. Perguson, D.E. Perkins, T. Petrie, C. Boxi, Rev. Sej. Instru. 59, 1879 (1988).

6. G.D. Hobbs and J.A. Wesson, Plasma Physics 8, 85 (1967).

7. G.D. Hobbs and J.A. Wesson, Culham Laboratory, Report CLM-R61 (1960).

8. G.D. Porter, Nucl. Fusion 22, 1279 (1982).

9. L.S. Hall, Nucl. Fusion 17, 681 (1977).

10. R. Choducs, Phys. Fluids 25, 1628 (1982).

11. V. Daybelge and B. Bein, Phys. Fluids 24, 1190 (1981).

12. C.F. Barnett, Oak Ridge National Laboratory Report ORNL-3113 (revised) (1961).

13. P.C. Stangeby and G.M. McCracken, Nucl. Fusion 30, 1225 (1990). 